\title{
High-repetition-rate combustion thermometry with two-line atomic fluorescence excited by diode lasers
}

\author{
Robin S. M. Chrystie, ${ }^{1}$ Iain S. Burns, ${ }^{3}$ Johan Hult, ${ }^{4}$ and Clemens F. Kaminski ${ }^{1,2, *}$ \\ ${ }^{1}$ Department of Chemical Engineering and Biotechnology, University of Cambridge, Pembroke Street, \\ Cambridge CB2 3RA, UK \\ ${ }^{2}$ SAOT-School of Advanced Optical Technologies, Max-Planck-Institute for the Science of Light, Division III, \\ Erlangen, Germany \\ ${ }^{3}$ Department of Chemical and Process Engineering, University of Strathclyde, Montrose Street G1 1XJ, UK \\ ${ }^{4}$ MAN Diesel A/S, Teglholmsgade 41, DK-2450 Copenhagen, Denmark \\ *Corresponding author: cfk23@cam.ac.uk
}

Received May 19, 2009; accepted June 15, 2009;

posted July 14, 2009 (Doc. ID 111548); published August 12, 2009

We report on kilohertz-repetition-rate flame temperature measurements performed using blue diode lasers. Two-line atomic fluorescence was performed by using diode lasers emitting at around 410 and $451 \mathrm{~nm}$ to probe seeded atomic indium. At a repetition rate of $3.5 \mathrm{kHz}$ our technique offers a precision of $1.5 \%$ at 2000 $\mathrm{K}$ in laminar methane/air flames. The spatial resolution is better than $150 \mu \mathrm{m}$, while the setup is compact and easy to operate, at much lower cost than alternative techniques. By modeling the spectral overlap between the locked laser and the probed indium lines we avoid the need for any calibration of the measurements. We demonstrate the capability of the technique for time-resolved measurements in an acoustically perturbed flame. The technique is applicable in flames with a wide range of compositions including sooting flames. (C) 2009 Optical Society of America

OCIS codes: $120.1740,140.2020,130.6010,140.0140,120.6780,300.6210$.

Temporally and spatially resolved flame measurements are important in an effort to improve the understanding of dynamic combustion phenomena. Although optical measurement techniques are capable of high spatial and temporal resolution, they are usually restricted to repetition rates of less than $10 \mathrm{~Hz}$, which does not permit dynamic events to be followed in real time. High-speed Rayleigh scattering is a notable exception [1]. It is limited by the requirement for constant Rayleigh cross sections in unburnt and burnt gases, and it requires expensive high-power lasers.

A simple yet highly accurate approach for measuring flame temperature at a point [2], or across a plane $[3,4]$, is two-line atomic fluorescence (TLAF) [5]. So far, most TLAF measurements have been performed at low repetition rates (of the order of $10 \mathrm{~Hz}$ ) except in an early demonstration by Dec and Keller [6] who used cw-dye lasers pumped by high-power argon-ion lasers for indium excitation at up to $5 \mathrm{kHz}$ repetition rates. Our group has introduced the use of extended cavity diode lasers (ECDLs) operating in the blue spectral region for TLAF measurements in flames $[7,8]$. Here, we demonstrate that ECDL excited TLAF can be used for highly accurate temperature measurement rates approaching $4 \mathrm{kHz}$. The technique's high spatial resolution $\left[\left(\begin{array}{cc}\sim 100 & \mu \mathrm{m}\end{array}\right)^{3}\right]$ and its low cost offer the potential for widespread use. The ECDL output is spectrally pure $(8 \mathrm{MHz}$ linewidth) and is stabilized by a simple method of locking the laser line to a reference wavelength. This eliminates problems caused by laser-mode noise on temperature measurements, which affected previous measurements [6]. It was also discovered that the indium absorption linewidth is of the order of 15 times larger than what had been assumed in [6] for the 410 $\mathrm{nm}$ transition at atmospheric pressure [7], which can cause systematic temperature errors. Furthermore, our single-detector approach [8] eliminates calibration error, since no temperature reference measurement is required.

The technique was validated in well-characterized laminar flames before continuously time-resolved measurements were then demonstrated in an acoustically forced, laminar Bunsen flame. In both flames, stoichiometric $\mathrm{CH}_{4}$ /air mixtures were used. The flames were seeded by passing the premixed gases through a nebulizer containing $0.03 \mathrm{M}$ aqueous $\mathrm{InCl}_{3}$ [9]. Indium was excited in the $5^{2} P_{1 / 2}-6^{2} S_{1 / 2}$ transition near $410 \mathrm{~nm}$ and the fluorescence signal from the $5^{2} P_{3 / 2}-6^{2} S_{1 / 2}$ transition near $451 \mathrm{~nm}$ was recorded by a photomultiplier tube (PMT, see Fig. 1) fitted with an interference filter. Subsequently, a second laser excited the $5^{2} P_{3 / 2}-6^{2} S_{1 / 2}$ transition and resonance fluorescence was detected near $451 \mathrm{~nm}$ on the same PMT.

Two custom designed ECDLs, whose design is fully described in [10], were used to excite the indium transitions in the linear regime, with output irradiances of approximately $17 \mathrm{~W} \mathrm{~cm}^{-2}(410 \mathrm{~nm})$ and $8 \mathrm{~W} \mathrm{~cm}^{-2}(451 \mathrm{~nm})$ at the measurement volume. To lock the wavelengths of the lasers near to the peaks of the indium transitions, weak reflections of the beams were directed through an indium hollow cathode lamp (HCL). The reference and transmitted powers were digitized (National Instruments PCI-6014), and a LabView program was used to lock the wavelength to the flank of one of the lamp lines by feedback control [8]. Figures 2(a) and 2(b) show the wavelength at which each laser was locked relative to the 


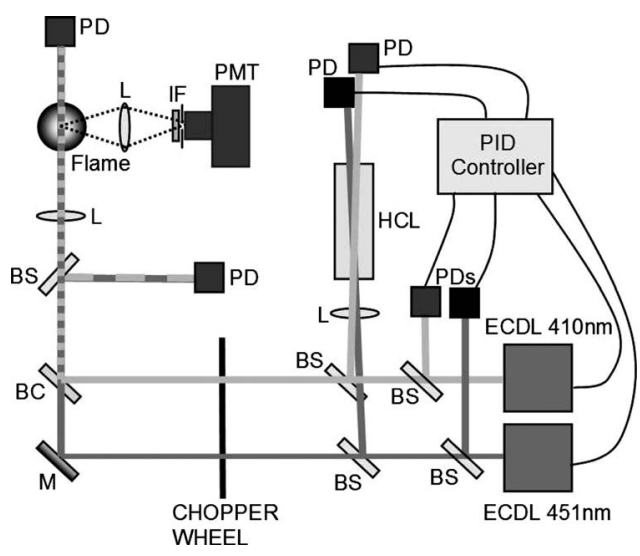

Fig. 1. Components of the setup comprise: photodiodes (PD), lenses (L), photomultiplier tube (PMT), interference filter (IF), beam splitters (BS), beam combiner (BC), mirror (M), hollow cathode lamp (HCL), and two ECDLs.

spectrum from the HCL.

Also shown in Fig. 2 are calculated fluorescence spectra that take account of the change in line intensity as a function of temperature, as well as the collisional shift and broadening. The ratio of normalized fluorescence signals, $R(T)=\left(F_{451} / I_{451}\right) /\left(F_{410} / I_{410}\right)$, at these locking positions is a sensitive and approximately linear function of temperature (here $F_{\lambda}$ $=$ fluorescence at excitation wavelength $\lambda, I_{\lambda}=$ laser irradiance). $R(T)$ can be modeled for the transitions and line shapes based on the collisional broadening and shift of the individual hyperfine components [9]. Temperature can thus be inferred from the experimentally determined values of $R(T)$ without any need for calibration.

A rotary chopper was used to switch rapidly between the two laser wavelengths; the rates of modulation (i.e., measurement speed) used in this study ranged from $500 \mathrm{~Hz}$ to $20 \mathrm{kHz}$. The beams were focused by a $300 \mathrm{~mm}$ lens onto the axis of the flame and indium fluorescence was imaged at right angles onto a $150 \mu \mathrm{m}$ pinhole situated in front of a filtered $(\Delta \lambda$ $=3 \mathrm{~nm}$ ) PMT (Hamamatsu, R3788).

During the initial experiments, the rate of modulation (measurement speed) was varied to investigate its influence on the precision of the determined temperature. For each modulation frequency the relative uncertainty was recorded at a range of heights above the flame front of the laminar flat flame. The uncertainty here is defined as the standard deviation of measured temperatures divided by its mean (100 repetitions). The relative uncertainty decreased with measurement height. Interestingly, near the flame front the largest uncertainties were observed at slower measurement speeds, with relative errors increasing nearly fourfold when decreasing measurement rates from $14 \mathrm{kHz}$ to $500 \mathrm{~Hz}$. We attribute this to fluctuations in indium concentration, caused by the seeding process. For long intervals between 410 and $451 \mathrm{~nm}$ excitations, indium concentrations in the measurement volume may change, resulting in signal ratios that do not correspond with the correct temperature. We believe this to be a consequence of the arrival of discrete solid particles of $\mathrm{InCl}_{3}$ at the
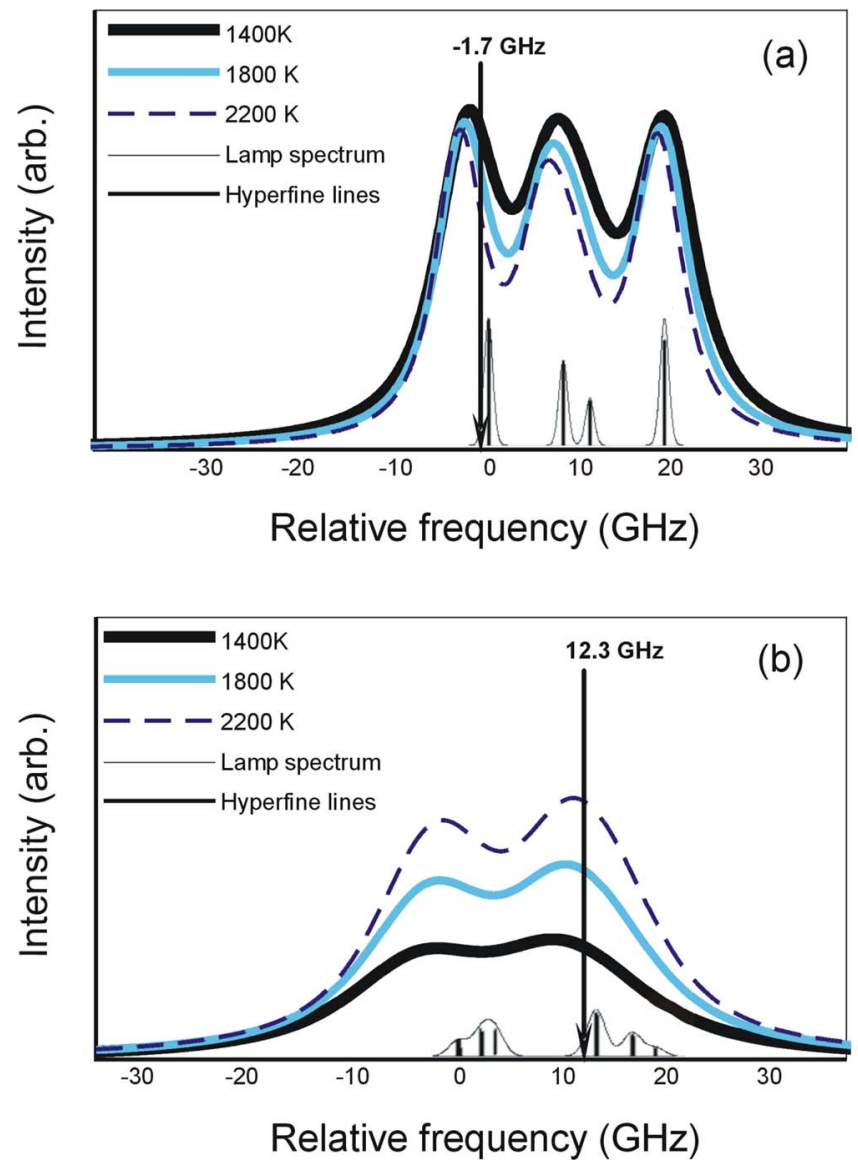

Fig. 2. (Color online) Curves represent indium spectra for excitation near (a) 410 and (b) $451 \mathrm{~nm}$ at various temperatures. Black, solid vertical lines denote locking positions of (a) -1.7 and (b) $12.3 \mathrm{GHz}$ on a relative frequency scale. The underlying peaks represent HCL spectra.

flame front, causing atomic indium to be liberated in discontinuous bursts. At increasing heights these concentration differences even out, leading to lower temperature uncertainties. There is thus an optimal measurement speed for the flames and setup employed here (around $14 \mathrm{kHz}$ ).

To demonstrate the capability of the technique to resolve dynamic flame features, measurements were conducted within a pulsed Bunsen flame described in [11]. Premixed reactants were passed through a tube of $10 \mathrm{~mm}$ diameter, and pressure perturbations were imposed on the flow by means of a loudspeaker situated $1 \mathrm{~m}$ upstream of the burner exit. The speaker was driven with pulses ( $2 \mathrm{~s}$ period). The resultant dynamic flame structure was visualized with an intensified CCD camera; see Figs. 3(a)-3(f). The time separation between subsequent images is $1 \mathrm{~ms}$. A small flame "globule" is pinched off and convects downstream following each pressure pulse. The point marked with a cross on the images denotes the position where time-resolved TLAF measurements were performed at a measurement speed of $14 \mathrm{kHz}$. The corresponding temperature trace is shown on the bottom pane (gray curve: raw data; black curve: average over four temperature samples, giving an effective sampling rate of $3.5 \mathrm{kHz}$.). A sample trace of the raw fluorescence signal is also shown in the inset (dotted box inside graph) for a snapshot in time over four 

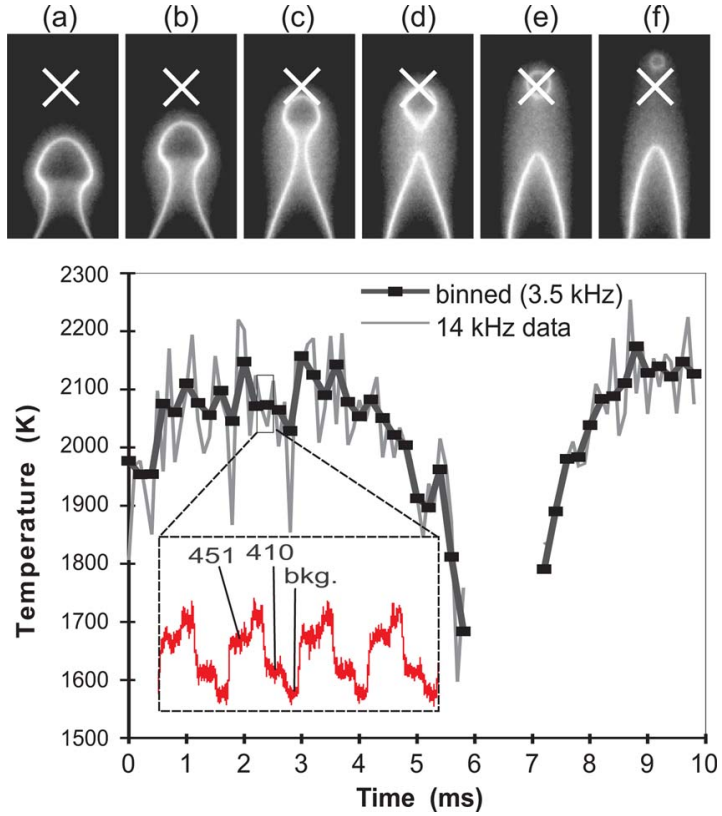

Fig. 3. (Color online) (a)-(f) of indium chemiluminescence show the flame front evolution after a pulse in steps of 1 $\mathrm{ms}$. The corresponding plot shows time-resolved temperatures at point $X$ in the flame along with the original fluorescence trace.

cycles at $14 \mathrm{kHz}$. The two signals at 410 and $451 \mathrm{~nm}$, along with the background, are shown for each cycle. At a $3.5 \mathrm{kHz}$ effective sampling rate-approximately the convective Batchelor frequency-our temperature uncertainty was $1.5 \%$ at $2000 \mathrm{~K}$, which compares very favorably with alternative, more complex, slower, and more expensive techniques [6]. Furthermore, the spatial resolution of our technique is good enough to resolve realistic Batchelor length scales $(200-500 \mu \mathrm{m})$ [1].

The temperature trace in Fig. 3 exhibits a pronounced dip in temperature when the laser beam is inside the flame globule where cool reactants are present. No data from the cool reactants within the flame bubble were available owing to the lack of free In atoms in this location. As the bubble passes through the measurement location the temperature rises once again. Note that the temperature profile is not symmetric in time, because the flame bubble is changing shape as it convects upstream (it becomes progressively smaller in time as the flame consumes the reactants inside). Such dynamic flame features cannot be resolved with static thermometry tech- niques, and there is scope here to collect data in turbulent systems for use in computational fluid dynamic code validation and development.

In conclusion, we have shown that low-power diode lasers can be used in combination with indium TLAF to measure spatially and temporally resolved temperature data quasi-continuously with favorable precision. The setup required is comparatively simple and economical compared to alternative techniques. We show that indium concentration fluctuations cause measurement errors at low sampling rates and that, for the conditions used in the present experiments, an optimal precision is obtained at a measurement speed of around $14 \mathrm{kHz}$ for locations near the flame front. We demonstrated quasi-continuous temperature measurements at an effective sampling rate of $3.5 \mathrm{kHz}$ repetition rates in dynamically perturbed flames with an associated precision of $1.5 \%$.

R. S. M. Chrystie was supported by an Engineering and Physical Sciences Research Council (EPSRC) CASE Studentship. I. S. Burns was supported by a Research Fellowship from St. John's College, Cambridge. C. F. Kaminski acknowledges sponsorship from the Leverhulme Trust, and J. Hult acknowledges support from the EPSRC. We are grateful to J. H. Frank for valuable advice.

\section{References}

1. G. H. Wang, N. T. Clemens, and P. L. Varghese, Appl. Opt. 44, 6741 (2005).

2. R. G. Joklik and J. W. Daily, Appl. Opt. 21, 4158 (1982).

3. J. Engström, J. Nygren, M. Aldén, and C. F. Kaminski, Opt. Lett. 25, 1469 (2000).

4. P. R. Medwell, Q. N. Chan, P. A. M. Kalt, Z. T. Alwahabi, B. B. Dally, and G. J. Nathan, Appl. Opt. 48, 1237 (2009).

5. G. Zizak, N. Omenetto, and J. D. Winefordner, Opt. Eng. 23, 749 (1984).

6. J. E. Dec and J. O. Keller, in Twenty-First Symposium on Combustion (The Combustion Institute, 1986), p. 1737.

7. J. Hult, I. S. Burns, and C. F. Kaminski, Opt. Lett. 29, 827 (2004).

8. J. Hult, I. S. Burns, and C. F. Kaminski, Proc. Combust. Inst. 30, 1535 (2005).

9. I. S. Burns, J. Hult, G. Hartung, and C. F. Kaminski, Proc. Combust. Inst. 31, 775 (2007).

10. J. Hult, I. S. Burns, and C. F. Kaminski, Appl. Opt. 44, 3675 (2005).

11. J. H. Frank and T. B. Settersten, Proc. Combust. Inst. 30, 1527 (2005). 